\title{
Classroom Searching System Based On Smart Mobile Phone
}

\author{
Dian $\mathrm{Gu}^{1 \mathrm{a}}$, Dongsong $\mathrm{Yan}^{2}$, Jiachun $\mathrm{Cai}^{3}$, Yijia Zheng ${ }^{4}$, Shuangrui Shi ${ }^{5}$, Luyang \\ $\mathrm{Liu}^{6}$
}

Jinan University , Zhuhai , Guangdong , China

a546780523@qq.com

Keywords: classroom searching, offline, Android, SQLite

Abstract. In order to solve the inconvenience of searching the location of classroom, we developed an offline classroom searching application based on smart mobile phone, which makes it possible to achieve the searching function in five campuses. This paper attaches importance to the installation of classroom data, the transition of different searching pages, the optimization of searching efficiency and data updating. This system doesn't need to connect to the Internet and impropriates few memory spaces. Moreover, the speed of searching is very fast. Therefore, it's a great tool that provides teachers and students with conveniences.

\section{Introduction}

Recently, applications based on Android platform are emerging, and have a large number of users. Most of the services that colleges provide for classroom information are based on PC, they can't be used whenever and wherever possible. Inevitably, the searching function of college classroom management system based on Internet is limited by time and space. However, most of the applications on mobile phones are based on the Internet, when someone doesn't have wifi or $4 \mathrm{G}$, he/her can't do the search.

As for course arranging in one semester, the use of classroom is settled, so we chose to install the classroom data to the phone in order to avoid using the Internet.

\section{System designing}

This off-line classroom searching system is based on Android platform, and classroom databases are according to the number of campuses. For example, my college has 5 different campuses, so we created 5 databases, including classroom information (location, time, course name, teacher, etc.) of each campus, with the length of 2000 5000.

The system will provide 3 different searching services focused on 3 kinds of users, because each kind has its unique need. Students can use it to search the use of classrooms, the time of courses and the classroom used by a certain teacher. Teachers can use it to search time and location of his/her courses. Managers can check the occupation situation of classrooms. Different kinds of users have different application interface, but they don't need to login. System managers update data every semester in order to keep the latest and most accurate searching result.

When using the application, first comes the welcome interface, then you can choose campus, finally you search your information. The flow chart of this system is shown in Fig.1.

(1) Query of Student: The system interface, including three dialog boxes of classroom query, time query and teacher name query, which respectively correspond to the three ways of the query. According to different searching logic, APP leads to the query results, showing the course name, classroom location, class time, and all the information of teachers.

(2) Query of Teacher: The system interface is the teacher name input box, the teacher enters the name and then APP jumps to the next interface, including the classroom and time dialog boxes for the users to query about all the class places and class time. After the teacher enters the information, APP jumps the interface again and displays the classroom information under the information condition. 
(3) Query of Manager: In the choice of management mode, the system interface jumps to the next interface which includes the classroom and time two dialog boxes, providing managers to query classroom using condition in a week or a certain time of all classroom using condition. Through this way managers can know the use of classroom allocation and using needs to facilitate the inspection, adjustment and arrangements.

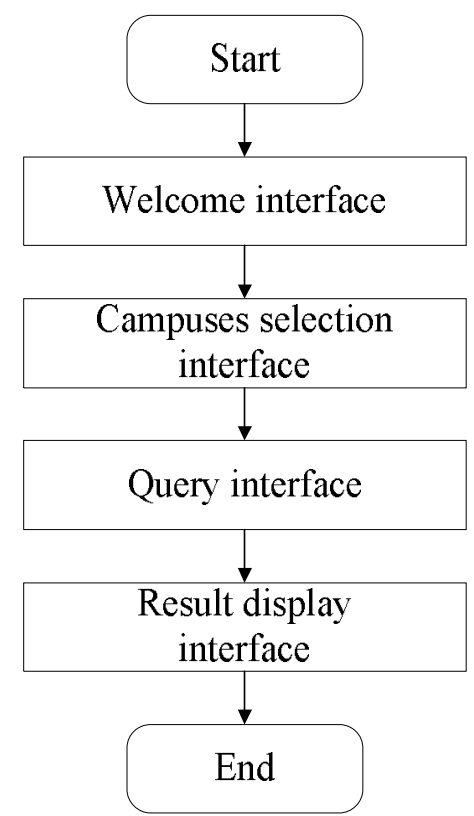

Fig.1 Flow chart of the application

\section{System implementation method}

\section{Database file design and installation}

Because of the geographical position, the school is divided into multiple campuses, but various campus classrooms are relatively independent, so as to establish the campus classroom database. By selecting a different campus to load the designated campus classroom data, not only to achieve the function of the campus query, and make the existence of a small call data file, which can make the system occupies small memory, running fast. In this way, we can save a lot of space on the use of mobile phone memory.

In the mounting of the APP to the mobile phone, also needs to be downloaded to the mobile phone on the classroom database, the establishment of data files need to have ROOT permissions in the mobile phone memory, but some mobile phone can not get ROOT permission, so we used to keep the way of the database file to solve the ROOT permission problem. The data file establishment and transfer method is firstly load the campus classroom information into the Assets directory, the installation program running on the mobile phone through the judgment after the implementation of the copy operation, get the information of the classroom contains ".db" data input stream, then the output stream will ".db" document written to the mobile phone SD card "data/data/com.example.search" folder. Without ROOT permission, we realized the establishment of classroom data file.

The following is a way to implement campus selection: set the five button and the corresponding listener firstly, listening to the click event, to trigger the campus selection function (such as our school consists of five campuses), and then define the string of five String type, representing five campuses. When the user selects one of the campuses, the button listener event is triggered, and the string representing the campus is shuttled through the intent to the next Activity. After choosing a good campus, we enter the SecondActivity, opening the string representing the campus information from the intent of FirstActivity. Next, according to the value of the campus, it chooses to open the campus classroom information containing the database, and to achieve the query. After the information query is completed, the results will be placed in the Array List, then load the Array List into the intent to carry out the data transfer between activities, realizing the Serializable interface. Finally, it extracts Array List in ThirdActivity, and displayed on the page. 


\section{The switching among the presenting pages}

Using Fragment, we can achieve the jump switching of teacher, classroom and time three pages. Fragment needs to achieve the communication among three activities. Take the FirstActivity as an example. After implementing the campus selection function, APP takes the jump between FirstActivity and Fragment: we use three fragments to correspond to the three button options for the teacher, classroom and time.

First based on the communication between FirstActivity and mainActivity, APP manages the Fragment by adding the Fragment Manager to the MainActivity and in the way of Radio Group. Fragment Manager controls the calls of each fragment by clicking on the corresponding button click event of the Fragment, such as calling the teacher's Fragment after clicking the teacher button click event.Radio Group can receive which fragment is called at any time and present the fragment on the main page while preventing possible conflicts between pages.

Above all, we realize the page switching between Fragment and FirstActivity . For users, they can see the required query page they want just by clicking the button on the APP. The communication of Fragment with other activities such as SecondActivity and ThirdActivity uses the same principle, they can be achieved in the same way.

\section{Implementation of query function}

\section{Implementation of multiple query methods}

The system provides three kinds of query methods: classroom query, teacher name query and the course time query. The normal flow of each method is as follows. First, the user input the information key into the system. Second, the system checks the input string and executes the query operation. Finally, the system returns the query result to the user interface. If any error occurs while querying, or the return data is empty, the system will perform other alternative flows. The query process is shown in Fig.2.

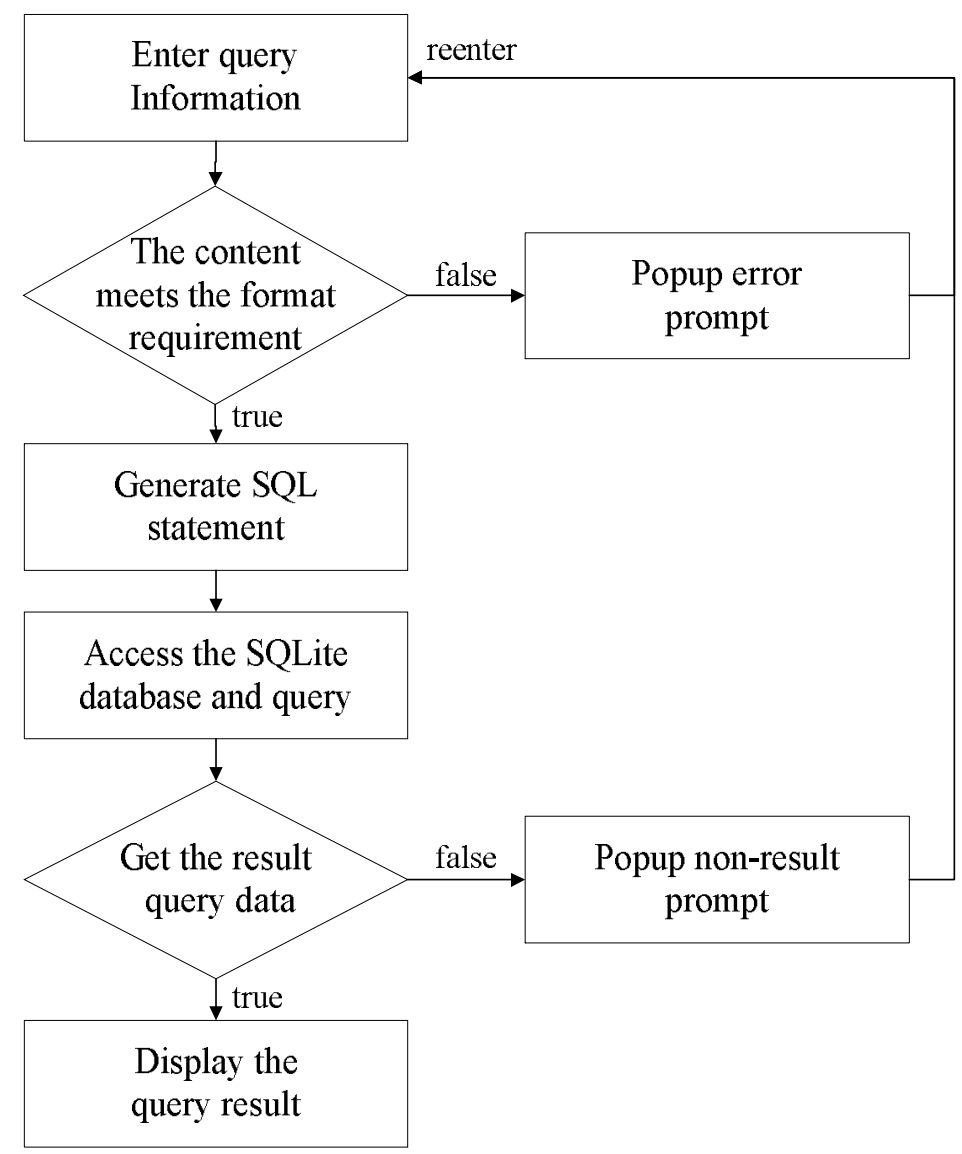

Fig.2 Flow chart of the query process

The implementation of all the three kinds of query methods using the fuzzy query, that is, to find all the courses data which containing the input key words. The system uses the 'like' predicate in SQL syntax to achieve the fuzzy query. The query statement is as follow: 
select * from List where the column name like '\%keyword\%'

The SQL statement above is used to query the corresponding column value that contains the key word, so as to achieve the function of fuzzy query.

\section{Query efficiency optimization}

Efficiency optimization includes two aspects: user input optimization and SQL query optimization. User input optimization can help users to enter the correct query string quickly, while the SQL query optimization can improve the searching efficiency of software to access the database.

User input optimization needs to increase fault tolerance control, that is, the appropriate way to deal with the user's input, which is not in the standard format. In this system, it is mainly embodied in the function module of course time query. Thinking about the user input string may have different formats, the system need to detect the input string and generate a string in line with a certain format for the query function. Meanwhile, the system should pop up an error message when a day number or class number cannot be detected.

The optimization of SQL query efficiency mainly includes the optimization the fuzzy query above. Due to the query using the 'like' predicate can only use the full table scan instead of the query with the index, which leads to low efficiency. Thus, in the specific application, equivalent query, single direction query and some other ways, which support the index query, can be used as alternative ways of traditional fuzzy query method. For example, the time query module can use the index query twice. The query process including the first query of courses data whose first class time is before the query time, and the second query of the courses data in the first search result, whose last course time is after the query time. So the two times index query can implement the same effect as the traditional fuzzy query with higher efficiency.

\section{The issue of data-updating}

The update is indispensable to the data in the databases of classroom in the new semester. When it comes to the privacy and the security, we have restricted access to the EAS (Educational

Administration System) due to the programming we made is off-line. The new data of the classroom, operated by maintenance staff, will be imported in software termed SQLite Expert Professional, after what a document with ".db" as a suffix will generate. The file then will build an apk packet once again. We are supposed to remind the users of the update just after we upload this apk to app store.

\section{Summary}

It is undeniable that the service of classroom-searching is an inevitable section in the informatization of Higher learning institution, especially when we consider its close association with students, professors as well as the administrators. This service is supposed to fulfill several valuable features in accuracy,convenience and instantaneity.In tearms of the drawbacks of the available classroom-searching system, we designed and actualized a off-line classroom searching system of great convenience,high efficienty and accurate timeliness. On one hand, this system can not only provide various routes to search the information of classrooms of several campuses, which considerably reduce the demand that users should import,but also enlarge the fault-tolerance compared with other systems ,in other words, it is more human-based.On the other hand,the achievement of the function of off-line searching offers much more conveniences along with solutions to both students and professors during their day-to-day life.It is worth mentioning that, only a few changes can make this system apply to various universities. The generality of this system has ability to enable itself to play an increasingly substantial role in this developing age. 


\section{References}

[1] Zhikui Zhou, Heling Wu: Database theory and Technology in Chinese. [M].1.Peking. Science Press (1999).

[2] Gang Li: Crazy Android in Chinese. [M].1.Peking. Electronic Industry Press (2011).

[3] Xiaozhen Ji: The Design and Implementation of Information Service System based on Android Platform in Chinese. [D].Huazhong University of Science and Technology (2013).

[4] Yang Yu: The Design and Implementation of Experiment Course Management System based on Android in Chinese. [D].University of Electronic Science and Technology of China (2014). 